\title{
Low-cost fabrication of optical waveguides, interconnects and sensing structures on all-polymer based thin foils
}

\author{
Maher Rezem*a , Christian Kelb ${ }^{\mathrm{a}}$, Axel Günther ${ }^{\mathrm{a}}$, Maik Rahlves ${ }^{\mathrm{a}}$, Eduard Reithmeier ${ }^{\mathrm{b}}$, Bernhard
} Roth $^{\mathrm{a}}$

${ }^{a}$ Hannover Centre for Optical Technologies, Leibniz Universität Hannover, Nienburger Strasse 17, Hanover, Germany; ${ }^{b}$ Institute of Measurement and Automatic Control, Leibniz Universität Hannover, Nienburger Strasse 17, Hanover, Germany

\begin{abstract}
Micro-optical sensors based on optical waveguides are widely used to measure temperature, force and strain but also to detect biological and chemical substances such as explosives or toxins. While optical micro-sensors based on silicon technology require complex and expensive process technologies, a new generation of sensors based completely on polymers offer advantages especially in terms of low-cost and fast production techniques. We have developed a process to integrate micro-optical components such as embedded waveguides and optical interconnects into polymer foils with a thickness well below one millimeter. To enable high throughput production, we employ hot embossing technology, which is capable of reel-to-reel fabrication with a surface roughness in the optical range. For the waveguide fabrication, we used the thermoplastic polymethylmethacrylate (PMMA) as cladding and several optical adhesives as core materials. The waveguides are characterized with respect to refractive indices and propagation losses. We achieved propagation losses are as low as $0.3 \mathrm{~dB} / \mathrm{cm}$. Furthermore, we demonstrate coupling structures and their fabrication especially suited to integrate various light sources such as vertical-cavity surface-emitting lasers (VCSEL) and organic light emitting diodes (OLED) into thin polymer foils. Also, we present a concept of an all-polymer and waveguide based deformation sensor based on intensity modulation, which can be fabricated by utilizing our process. For future application, we aim at a low-cost and high-throughput reel-to-reel production process enabling the fabrication of large sensor arrays or disposable single-use sensing structures, which will open optical sensing to a large variety of application fields ranging from medical diagnosis to automotive sensing.
\end{abstract}

Keywords: Hot embossing, optical waveguide, polymer optics, integrated photonics

\section{INTRODUCTION}

Because of numerous advantages compared to conventional electronic sensors, micro optical systems are being investigated by many research groups around the world. Taking advantages of the constant advances in microelectronics fabrication techniques, silicon photonics has applications in several fields, such as optical communication, lasers and sensor technology [1]. However, the silicon based fabrication techniques used are cost and time intensive. The need for low cost micro-optic components can be met through the use of polymer materials. In fact, polymer materials are less expensive compared to silicon based materials. Furthermore, polymers can potentially be processed using high throughput reel-to-reel techniques, which further lower the fabrication costs of all-polymer based micro-optical components.

In this work, we present our recent research in the field of all-polymer based foil-integrated micro-optical systems. First, we discuss low-cost fabrication of low-loss planar multimode optical waveguides based on hot embossing, doctor blading and UV curing. Then, we investigate the fabrication of low-loss interconnects and coupling structures, which enables the integration of optical sources, detectors and fibers. Finally, we discuss an optical strain sensor concept, which can be manufactured using the presented fabrications processes. In the future, the presented fabrication process will be implemented in a reel-to-reel process which will increase the throughput rates for integrated waveguide production. Application scenarios include mass-market fabrication of micro-optical sensor devices ranging from temperature sensors to disposable lab-on-chip sensor devices.

*maher.rezem@hot.uni-hannover.de; phone $0049511 \quad 76217303$; fax 0049511 76217909; hot.uni-hannover.de

Smart Photonic and Optoelectronic Integrated Circuits XVIII, edited by Sailing He, El-Hang Lee, Louay A. Eldada, Proc. of SPIE Vol. 9751, 975112 - (c) 2016 SPIE · CCC code: 0277-786X/16/\$18 · doi: 10.1117/12.2213182 


\section{ALL-POLYMER MULTIMODE OPTICAL WAVEGUIDES}

In this section, we discuss the low cost fabrication process of all-polymer based multimode optical waveguides. Then, the characterization results of the fabricated waveguides are presented. Finally, we discuss a potential reel-to-reel implementation of the presented process, which leads to lower costs and higher production throughput.

\subsection{Fabrication}

In this work, we fabricate all-polymer multimode optical waveguides based on three fabrication steps, consisting of hot embossing of the cladding material, application of the core layer through doctor blading and UV-curing of the core material. The presented process is summarized in figure 1.

a)

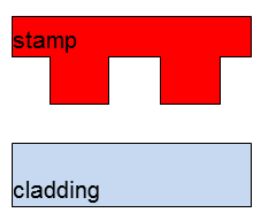

b)
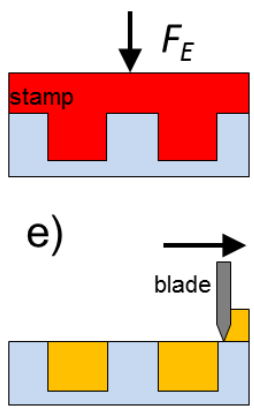

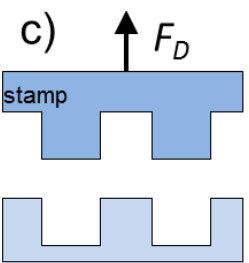

f)

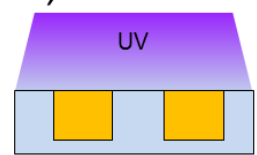

d)

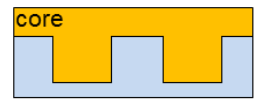

Figure 1. Summary of the presented optical waveguide fabrication process: (a) heating, (b) embossing, (c) cooling and demolding, (d) deposition of the core material, (e) doctor blading process, (f) UV-curing of the core material

For the first hot embossing step, we chose the thermoplastic polymethylmethacrylate (PMMA) as substrate material. Hot embossing consists of transferring the surface structures of a master stamp onto the polymer substrate, which represents the replica of the stamp. We performed this process using a commercial hot embossing machine (HEX03, Jenoptik Mikrotechnik $\mathrm{GmbH}$, Germany). The machine has a plate-to-plate configuration and can deliver embossing temperatures of up to $300^{\circ} \mathrm{C}$ and embossing forces of up to $300 \mathrm{kN}$. As a master stamp, we used a silicon wafer, which was fabricated by Micromotive $\mathrm{GmbH}$. The silicon wafer was patterned through photolithography, followed by a deep reactive-ion etching step. In order to reduce the scattering losses of the optical waveguides, the stamp surface roughness should be less than a few $\mathrm{nm}$. Therefore, the silicon wafer was finally processed with a plasma etching step. Using a laser scanning microscope (VK X100, Keyence, Japan), the average surface roughness Ra of the cladding was measured. We obtained Ra values of under $6 \mathrm{~nm}$. The fabricated stamp includes straight and bent rib-structures having a height and width of $25 \mu \mathrm{m}$, respectively, that can be used to fabricate straight and bent optical waveguides.

The hot embossing can be divided in four sub-steps, which consist of the heating, the embossing, the cooling and the

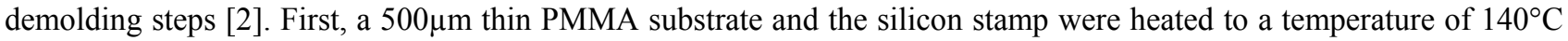
during the heating step. At this temperature, the used thermoplastic reaches its rubbery state [3] and is suited for pattern replication. During the following embossing step, an embossing force of $4 \mathrm{kN}$ was applied between the stamp and the PMMA substrate and maintained for 2 minutes, thus transferring the stamp structures on the PMMA surface. The force was maintained during the subsequent cooling step, where the stamp and the thermoplastic were cooled to a temperature of $50^{\circ} \mathrm{C}$. In the final demolding step, the embossing force was released and the stamp and PMMA were separated manually. The obtained result consists of a PMMA cladding that includes trench structures.

Following the hot embossing step, the doctor blading step which consists of depositing a liquid uncured waveguide core material on the cladding surface and removing the superfluous material using a razor blade was applied. Although other processes can be used to apply the core material, we opted for the doctor blading method because of its compatibility with reel-to-reel processes and its resulting thin residual layers. After applying the waveguide core material, we performed the final fabrication step, which consists of the UV-curing of the material. In this work, we investigated three different low-cost optical adhesives consisting of NOA68 (Norland, USA), OG198-54 (Epotek, USA) and OG142 (Epotek, USA). These materials were cured under a UV-lamp emitting at a wavelength of $365 \mathrm{~nm}$. 


\subsection{Characterization}

As a first characterization step, we photographed the cross-section of fabricated waveguides using an optical microscope. Figure 2 shows an example of such a waveguide cross-section, which was fabricated using PMMA and OG142.

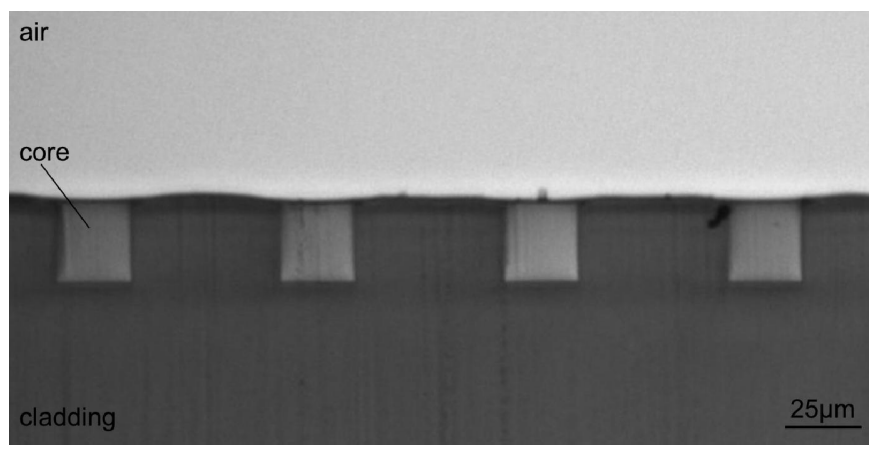

Figure 2. Cross-section of fabricated multimode waveguides using PMMA and OG142.

Furthermore, we analyzed the refractive index of the core materials utilized based on the refracted near-field method. Therefore, we employed a refractive index profiler (Rinck Elektronik $\mathrm{GmbH}$, Germany) and measured the refractive index of the waveguide core materials at a temperature of $20^{\circ} \mathrm{C}$ and at a wavelength of $638 \mathrm{~nm}$. Table 1 summarizes the obtained index values.

Table 1. Measured refractive index of the waveguide core materials used.

\begin{tabular}{|c|c|}
\hline $\begin{array}{c}\text { Waveguide core } \\
\text { material }\end{array}$ & $\begin{array}{c}\text { Measured refractive index } \\
\left(\mathbf{2 0} \mathbf{C}^{\mathbf{C}} \mathbf{6 3 8} \mathbf{~ n m}\right)\end{array}$ \\
\hline OG142 & 1.568 \\
\hline OG198-54 & 1.524 \\
\hline NOA68 & 1.525 \\
\hline
\end{tabular}

Next, we measured the propagation losses of the fabricated optical waveguides at the wavelengths of 633 and $850 \mathrm{~nm}$ following the so-called cut-back method [4]. Therefore, we measured the insertion losses of multiple waveguides, which have different lengths. Based on the measured values and using linear regression, we determined the propagation losses of the waveguides in $\mathrm{dB} / \mathrm{cm}$. For the characterization at a wavelength of $633 \mathrm{~nm}$, we used a helium-neon laser (25-LHP991, Melles Griot, USA) for input. Using a microscope objective, the laser beam was focused on the waveguide input facets. For the characterization at a wavelength of $850 \mathrm{~nm}$, we used a fiber coupled laser diode (MCLS1-850, Thorlabs, USA). For both wavelengths, the sample was positioned using a 3-axis precision stage. An output fiber was then buttcoupled on the waveguide output facets using a 5 -axis precision stage. The $50 \mu \mathrm{m}$ output fiber was connected to a photodiode power sensor (S151C, Thorlabs, USA), which measures the optical power that enables determination of the insertion loss of the respective waveguide. The measured propagation losses of the different core materials are summarized in table 2 .

Table 2. Measured propagation losses of the fabricated waveguides for different materials and wavelengths.

\begin{tabular}{|c|c|c|}
\hline $\begin{array}{c}\text { Waveguide core } \\
\text { material }\end{array}$ & $\begin{array}{c}\text { Propagation loss @633nm } \\
{[\mathbf{d B} / \mathbf{c m}]}\end{array}$ & $\begin{array}{c}\text { Propagation loss @850nm } \\
{[\mathbf{d B} / \mathbf{c m}]}\end{array}$ \\
\hline OG142 & 2.56 & 1.05 \\
\hline OG198-54 & 0.97 & 0.31 \\
\hline NOA68 & 0.74 & 0.81 \\
\hline
\end{tabular}




\subsection{Potential reel-to-reel implementation}

As mentioned above, the presented waveguide fabrication process is based on hot embossing, doctor blading and UVcuring. These three steps are compatible with reel-to-reel processes. Having as a goal the increase of the fabrication scale and throughput, we propose a reel-to-reel optical waveguide fabrication process on polymer foils, which is summarized in figure 3: An input roll of cladding material feeds a reel-to-reel hot embossing system, which is composed of an infrared heater and a roll mold. The embossed thermoplastic cladding foil is then transferred to a roll coater that deposits the core material. Next, the excess core material is removed using doctor blading and the foil is transferred under a UVcuring system before reaching the output roll. This proposed reel-to-reel process is based on exactly the same waveguide fabrication steps of the presented plate-to-plate process. However, using a reel-to-reel method largely increases the fabrication scale and throughput and enables the fabrication of large sensor arrays or low-cost disposable sensors.

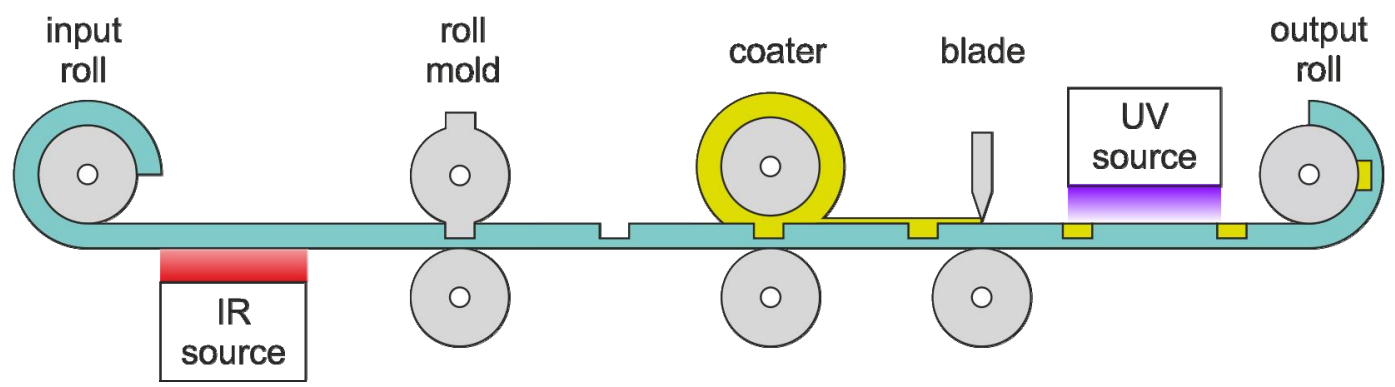

Figure 3. Proposed reel-to-reel implementation of the optical waveguide fabrication process.

\section{COUPLING STRUCTURES}

Besides optical waveguides, low loss coupling structures and interconnects represent an important building block of integrated polymer optical sensors. The purpose of these structures is to efficiently couple light in and out of the waveguides. Depending on the used light sources and detectors, different types of coupling structures come into question, such as optical interconnects between micro-optical components, mirror couplers and grating couplers.

For the fabrication of interconnects, self-written waveguides represent an attractive solution due to their low cost and low losses. For the present work, we developed a five step interconnect fabrication process [5], which is based on selfwritten waveguides and was tested as a fiber-to-fiber interconnect. In the first fabrication step, 5-axis precision stages were used to butt-couple two optical fibers. An UV curable monomer was then deposited on the gap between both waveguides. After separating the fibers, a laser source emitting at $405 \mathrm{~nm}$ (Thorlabs, MCLS 1-405-30) was connected to the launching fiber and switched on. Starting from the facet of the launching fiber, polymerization of the monomer propagates to the receiving fiber due to a self-focusing effect of the beam inside the monomer and its result is a solid core material surrounded by a liquid cladding. Finally, the cladding is cured through a flood exposure at a wavelength of $254 \mathrm{~nm}$. An example of such fabricated interconnect is shown in figure 4. The measured propagation loss of the selfwritten waveguides equals $0.8 \mathrm{~dB} / \mathrm{cm}$. However, since the distance between the two coupled fibers is very short, interconnect losses of $0.01 \mathrm{~dB}$ were measured [6].

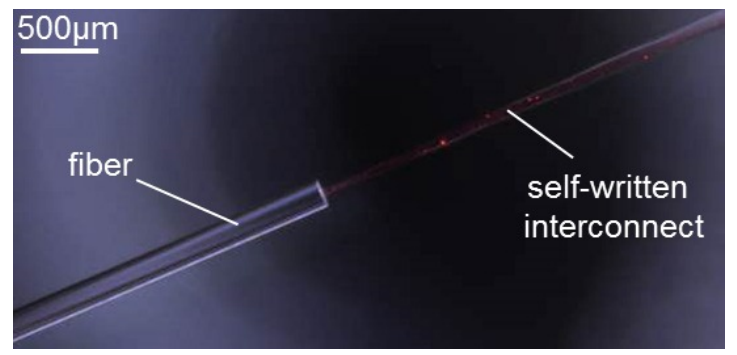

Figure 4. Microscope image of a fabricated self-written interconnect.

The presented interconnects are suited for coupling waveguides with fibers or side emitting laser diodes. For vertical emitting laser diodes (VCSEL), light coupling through optical gratings or mirrors represents a better alternative. For the case of mirror couplers, a $45^{\circ}$ slope is generated in an optical waveguide, as shown in figure 5 (a). Light, which 
propagates orthogonal to the waveguides, is then mirrored on the core-air interface through total internal reflection and starts propagating in the waveguide core. For this work, the slope was fabricated through a slotting process. A diamond tool, which has an opening angle of $90^{\circ}$, was used in a precision milling machine to produce the desired structure on the waveguide surface. The fabrication result is shown in figure 5 (b).

a)

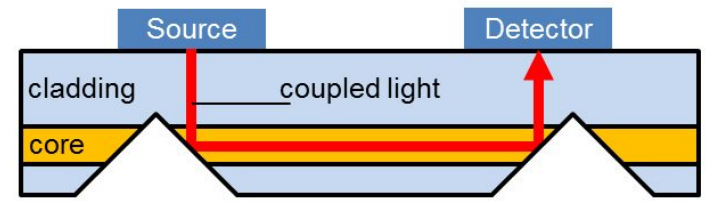

b)

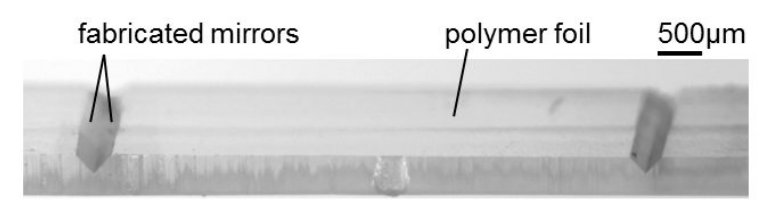

Figure 5. Light coupling through a mirror structure: a) Sketch of the concept, b) Result of slotting fabrication process.

For the case of grating couplers, a grating structure is produced on the waveguide core surface. Light propagating orthogonal to the surface is then diffracted on the grating surface. The goal is to choose the right grating dimensions (height, period), which enables coupling of the light into the waveguide core. This concept is feasible using transmission or reflection gratings, as shown in figure 6 (a). As a preliminary work for the implementation of this concept, we investigated the fabrication of grating structures through hot embossing. A hot embossed grating structure is shown is figure $6(b)$.

a)

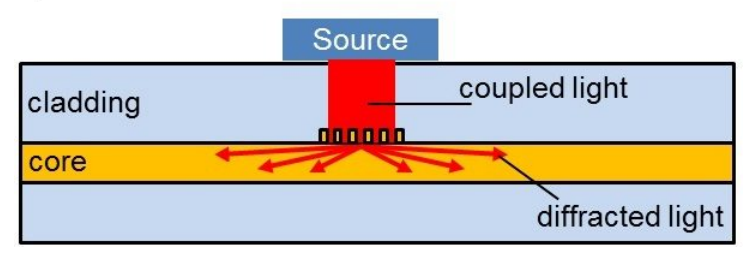

b)

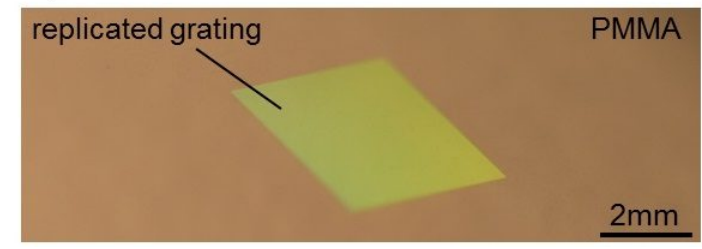

Figure 6. Light coupling through a grating structure: a) Sketch of the concept, b) Hot embossed grating structure.

\section{OPTICAL STRAIN SENSOR}

The presented waveguide and coupling structure fabrication processes enable the manufacturing of all-polymer integrated micro-optical systems, which can be used in various applications, such as sensor technology and optical communications. As an application example, we present an optical planar strain sensor, which is composed of two sets of aligned waveguide arrays, as shown in figure 7. The waveguide arrays are separated by an elongation zone. The function of the device is based on the analysis of the optical coupling efficiency between both waveguide arrays. A force, which is applied between both waveguide arrays, leads to a change in the length of the elongation zone. This leads to a variation in the coupled optical power into the output array. In an earlier work, the simulations for the sensor concept were discussed [7]. Furthermore, a prototype using commercial polymer optical fibers was presented [8]. The next steps of our work consist of the fabrication of this strain sensor using the presented waveguide fabrication techniques.

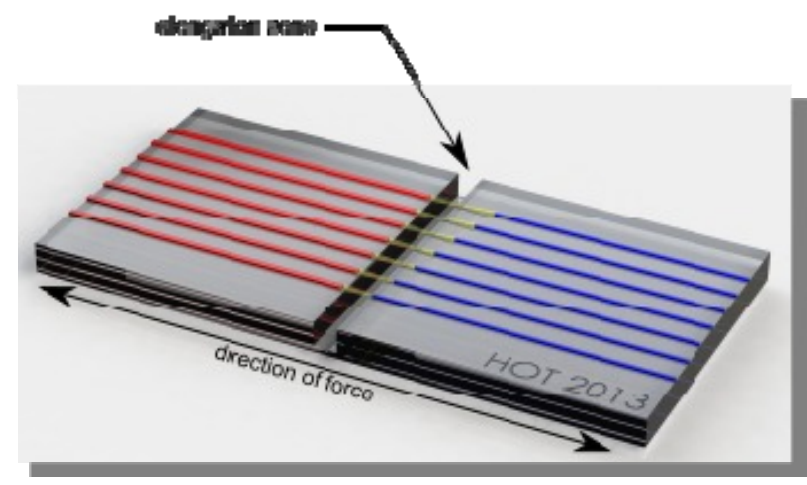

Figure 7. Sketch of the concept of the planar optical strain sensor based on intensity modulation [7]. 


\section{CONCLUSION}

In this paper, we presented a fabrication process of all-polymer multimode optical waveguides, which is based on hot embossing, doctor blading and UV curing. We used the thermoplastic PMMA as a waveguide cladding and several lowcost optical adhesives as core materials. We characterized the fabricated waveguides with respect to refractive index and propagation losses. We also discussed the implementation of the presented process in a reel-to-reel configuration, thus increasing its scale and throughput. Furthermore, we presented different light coupling solutions to integrate discrete micro-optical components on the fabricated polymer foils. We investigated the fabrication of interconnects using selfwritten waveguides and reached coupling losses of $0.01 \mathrm{~dB}$. Additionally, we discussed light coupling from vertical emitting sources through the use of mirror couplers and grating couplers and light coupling from horizontal sources and fibers and presented our first experimental results. Finally, we presented a concept of an optical strain sensor, which is based on intensity modulation and can be implemented through the presented fabrication processes. The long-term goal of the presented research is the implementation of a low-cost and high-throughput production process, which enables the fabrication of all-polymer foil-integrated sensor arrays and disposable sensing structures, which can be used in a large variety of sensing applications.

\section{ACKNOWLEDGMENTS}

This work was funded by the German Research Foundation (DFG) in the framework of the collaborative research center "PlanOS".

\section{REFERENCES}

[1] Ye, W. N., Xiong, Y., "Review of silicon photonics: history and recent advances," Journal of Modern Optics 60(16), 1299-1320 (2013).

[2] Worgull, M. [Hot Embossing: Theory and technology of microreplication], William Andrew, United Kingdom, (2009)

[3] Lan, S., Lee, H. J., Lee, S. H., Ni, J., Lai, X., Lee, H. W., Song, J. H., Lee, M. G., "Experimental and numerical study on the viscoelastic property of polycarbonate near glass transition temperature for micro thermal imprint process," Materials \& Design 30(9), 3879-3884 (2009).

[4] Tong, X. C. [Advanced Materials for Integrated Optical Waveguides], Springer, Switzerland, (2014)

[5] Günther, A., Petermann, A. B., Gleissner, U., Hanemann, T., Reithmeier, E., Rahlves, M., Wollweber, M., Morgner, U., Roth, B., "Cladded self-written multimode step-index waveguides using a one-polymer approach," Optics Letters 40(8), 1830-1833 (2015).

[6] Rezem, M., Günther, A., Petermann, A. B., Kelb, C., Rahlves, M., Wollweber, M., Roth, B., "Production and characterization of all-polymer based optical waveguides and interconnects," Proc. DGAO 116, (2015).

[7] Kelb, C., , Reithmeier, E., Roth, B., "Planar integrated polymer-based optical strain sensor," Proc. SPIE 8977, 89770 Y (2014).

[8] Kelb, C., Rahlves, M., Reithmeier, E., Roth, B., "Realization and Performance of an All-Polymer Optical Planar Deformation Sensor," IEEE Sensors Journal 15(12), 7029-7035 (2015). 